\title{
CYCLIC SUBGROUPS OF ORDER 4 IN FINITE 2-GROUPS
}

\author{
ZVONIMIR JANKO \\ University of Heidelberg, Germany
}

\begin{abstract}
We determine completely the structure of finite 2-groups which possess exactly six cyclic subgroups of order 4 . This is an exceptional case because in a finite 2-group is the number of cyclic subgroups of a given order $2^{n}$ ( $n \geq 2$ fixed) divisible by 4 in most cases and this solves a part of a problem stated by Berkovich. In addition, we show that if in a finite 2 -group $G$ all cyclic subgroups of order 4 are conjugate, then $G$ is cyclic or dihedral. This solves a problem stated by Berkovich.
\end{abstract}

\section{INTRODUCTION AND KNOWN RESULTS}

For a finite 2-group $G$ and a fixed integer $n \geq 1$ we denote with $c_{n}(G)$ the number of cyclic subgroups of order $2^{n}$. The starting point are the following results of Y. Berkovich. Suppose that a finite 2-group $G$ is neither cyclic nor of maximal class. Then $c_{1}(G) \equiv 3(\bmod 4)$ and if $n \geq 2$, then $c_{n}(G)$ is even (Berkovich [2, Theorem 1.17]). If in addition $G$ is nonabelian and $n \geq 3$, then $c_{n}(G) \equiv 0(\bmod 4)$ unless $G$ is an $L_{2}$-group or a $U_{2}$-group (Berkovich [1] and [2, Corollary 18.7]).

We shall use freely the above two results and we consider here only finite 2 -groups with a standard notation. In addition, a 2-group $G$ is called an $L_{2}$ group if $\Omega_{1}(G) \cong E_{4}$ is a four-subgroup and and $G / \Omega_{1}(G)$ is cyclic of order $\geq 4$. We note that an $L_{2}$-group $G$ is either abelian of type $\left(2,2^{m}\right), m \geq 3$, or

$$
G \cong M_{2^{m+1}}=\left\langle a, b \mid a^{2^{m}}=b^{2}=1, m \geq 3, a^{b}=a^{1+2^{m-1}}\right\rangle .
$$

A 2-group $G$ is called a $U_{2}$-group (with respect to $R$ ) if $G$ possesses a normal four-subgroup $R$ such that $G / R$ is a group of maximal class (i.e., $G / R$ is dihedral $D_{2^{n}}$, generalized quaternion $Q_{2^{n}}$ or semi-dihedral $S D_{2^{n}}$ ) and whenever

2000 Mathematics Subject Classification. 20D15.

Key words and phrases. Finite 2-groups, 2-groups of maximal class, minimal nonabelian 2-groups, $L_{2}$-groups, $U_{2}$-groups. 
$T / R$ is a cyclic subgroup of index 2 in $G / R$, then $\Omega_{1}(T)=R$. It is easy to see that the four-subgroup $R$ is uniquely determined. All $U_{2}$-groups are completely classified in Janko [3, section 6]. Finally, for a 2-group $G$, we define $\Omega_{n}^{*}(G)=\left\langle x \in G \mid o(x)=2^{n}\right\rangle$.

Here we shall consider the exceptional cases, where in a 2 -group $G$ we have $c_{2}(G) \equiv 2(\bmod 4)$. If $c_{2}(G)=2$, then such 2 -groups $G$ are already known (see Janko [4, Proposition 1.4, Theorems 5.1 and 5.2]). If $c_{2}(G)=6$, then such 2-groups $G$ are determined only in the special case where $\left|\Omega_{2}^{*}(G)\right|=2^{4}$. Such 2-groups $G$ with $|G|>2^{4}$ are determined by Janko [4, Theorem 2.1] when $\left|\Omega_{2}(G)\right|=2^{4}$ (since in that case $\Omega_{2}(G) \cong Q_{8} \times C_{2}$ or $\left.\Omega_{2}(G) \cong C_{4} \times C_{4}\right)$ and by Janko [3, Theorem 4.1] when $\left|\Omega_{2}(G)\right|>2^{4}$. In this paper we shall classify 2-groups $G$ with $c_{2}(G)=6$ and $\left|\Omega_{2}^{*}(G)\right|>2^{4}$. First we show that we must have $\left|\Omega_{2}^{*}(G)\right|=2^{5}$ and we get three possibilities for the structure of $\Omega_{2}^{*}(G)$ (Theorem 2.6). The corresponding 2-groups $G$ are determined up to isomorphism in Theorem 2.7. The general case, where $c_{2}(G) \equiv 2(\bmod 4)$ and $c_{2}(G) \geq 10$ is very difficult and is still open.

At the end we consider 2-groups $G$ which possess only one conjugate class of cyclic subgroups of order 4 and we show that in that case $G$ has only one cyclic subgroup of order 4 and therefore $G$ is either cyclic or dihedral (Theorem 3.1).

For convenience we state another known result which is of special importance in the proof of Theorem 2.6.

Proposition 1.1. (see [3, Proposition 1.2]) Let $K$ be a 2-group of order $>2^{3}$ possessing exactly two cyclic subgroups $U_{1}, U_{2}$ of order 4 and assume that neither of them is a characteristic subgroup of $K$. Then one of the following holds:

(i) $K \cong D_{8} \times C_{2}$ (of order $2^{4}$ ) with $\Phi(K)=U_{1} \cap U_{2} \cong C_{2}$;

(ii) $K$ is a uniquely determined group of order $2^{5}$ with $\Phi(K)=\left\langle U_{1}, U_{2}\right\rangle \cong$ $C_{4} \times C_{2}$.

\section{NeW ReSults FOR $c_{2}(G)=6$}

In what follows $G$ will denote a 2-group with $c_{2}(G)=6$ and $H=\Omega_{2}^{*}(G)$ is of order $>2^{4}$. Since $H$ has exactly six cyclic subgroups of order $4, H$ is neither cyclic nor a group of maximal class. It follows that $H$ possesses a $G$-invariant four-subgroup $W$ (see [6, Proposition 2.19]).

LEMMA 2.1. If a cyclic subgroup $V$ of order 4 in $G$ normalizes another cyclic subgroup $U$ of order 4 , then $U$ normalizes $V$ and $U V \cong C_{4} \times C_{2}$ or $U V \cong Q_{8}$.

Proof. First suppose $U \cap V=\{1\}$. Then $|U V|=2^{4}$ and $(U V)^{\prime}<U$ and we have either $U V=U \times V \cong C_{4} \times C_{4}$ or $(U V)^{\prime} \cong C_{2}$ in which case $U V$ is a metacyclic minimal nonabelian group of order $2^{4}$ and exponent 4 . In 
any case, $c_{2}(U V)=6$ and so $U V=H=\Omega_{2}^{*}(G)$, contrary to our assumption that $|H|>2^{4}$. Thus, $U \cap V \cong C_{2}$ and so $|U V|=2^{3}$. In this case, $U$ also normalizes $V$ and the only possibilities are $U V \cong C_{4} \times C_{2}$ or $U V \cong Q_{8}$.

Lemma 2.2. Suppose that $H=\Omega_{2}^{*}(G)$ contains a quaternion subgroup $Q \cong Q_{8}$. Then $|H|=2^{5}$ and we have the following two possibilities:

(a) $H \cong Q_{8} * Q_{8}$;

(b) $H \cong Q_{16} * C_{4}$.

Proof. First we determine the structure of $S=W Q$, where $W$ is a normal four-subgroup in $H$ and $|W \cap Q| \leq 2$. Let $z$ be an involution in $W \cap Z(S)$. If $z \notin Q$, then $c_{2}(Q \times\langle z\rangle)=6$ and therefore $Q \times\langle z\rangle=\Omega_{2}^{*}(G)=H$, a contradiction. Hence $Q \cap W=\langle z\rangle \cong C_{2},|S|=2^{4}$, and $[W, Q]=\langle z\rangle$. This gives $S=Q *\langle v\rangle$, where $\langle v\rangle \cong C_{4}$ and $\langle v\rangle \cap Q=\langle z\rangle$. We have $c_{2}(S)=4$, $\langle v\rangle=Z(S)$ and since all elements in $S-(Q \cup\langle v\rangle)$ are involutions, $Q$ is a unique quaternion subgroup of $S$ and therefore $Q$ is characteristic in $S$.

Assume that $S$ is not normal in $H$ and set $K=N_{H}(S)$ so that $|K: S| \geq 2$ and $|H: K| \geq 2$. Let $M$ be a subgroup of $H$ containing $K$ so that $|M: K|=2$ and take an element $m \in M-K$. Since $m$ normalizes $W$, we have $Q^{m} \neq Q$, $Q^{m} \not \leq S$ and $Q^{m} \leq K$. We have $\left|Q^{m} \cap S\right| \leq 4$ and so $Q^{m}-S$ contains at least four elements of order 4 . It follows that $c_{2}(K)=6$. But $\Omega_{2}^{*}(G)=H$ and so there are elements of order 4 in $H-K$, a contradiction. We have proved that $S$ is normal in $H$ and so $Q$ and $Z(S)=\langle v\rangle$ are normal in $H$. Since $c_{2}(S)=4$, we have exactly four elements of order 4 in $H-S$. Set $C=C_{H}(Q)$ so that $C$ is normal in $H$ and $|H:(Q C)| \leq 2$ (since $\left.\operatorname{Aut}(Q) \cong S_{4}\right)$.

If there is an involution $u$ in $C-\langle v\rangle$, then $c_{2}(Q \times\langle u\rangle)=6$ and $Q \times\langle u\rangle=H$, a contradiction. Hence $C$ is either generalized quaternion or cyclic. In the first case (since $C-\langle v\rangle$ can contain at most four elements of order 4 ), $C \cong Q_{8}$, $Q C \cong Q_{8} * Q_{8}, c_{2}(Q C)=6$ and therefore $Q C=H$ is an extraspecial group of order $2^{5}$ (case (a) of our lemma).

We may assume that $C$ is cyclic so that $|H:(Q C)|=2$ because $H-S$ must contain exactly four elements of order 4 . Since $H / C \cong D_{8}$, there is $x \in H-(Q C)$ such that $x^{2} \in C$ and $x$ induces an involutory outer automorphism on $Q$. There are elements $a, b \in Q$ such that $\langle a, b\rangle=Q, a^{x}=a^{-1}$ and $b^{x}=a b$.

Suppose that $\langle x, C\rangle$ is cyclic so that $\langle x, C\rangle=\langle x\rangle$. If $o(x) \geq 16$, then there are no elements of order 4 in $H-(Q C)$, a contradiction. Hence $o(x)=8$ so that we may assume that $x^{2}=v$. In this case all eight elements $l x(l \in S-\langle a, v\rangle)$ in $H-S$ are of order 4 , a contradiction. Hence $\langle x, C\rangle$ is noncyclic.

Assume that $\langle x, C\rangle$ is abelian or $\langle x, C\rangle \cong M_{2^{m}}, m \geq 4$, so that in both cases we may assume that $x$ is an involution centralizing $\langle v\rangle$. We have $o(x v)=o(x v a)=4$ and we see that $c_{2}(S\langle x\rangle)=6$ and so we get $H=S\langle x\rangle=\langle Q, x v\rangle *\langle v\rangle$, where $\langle Q, x v\rangle \cong Q_{16}$ and $H \cong Q_{16} * C_{4}$ (case (b) of our lemma). 
Assume that $\langle x, C\rangle \cong Q_{2^{n}}$ or $\langle x, C\rangle \cong S D_{2^{n}}$. In both cases we may assume that $x^{2}=z$ and $\langle v, x\rangle \cong Q_{8}$ since $Q_{8}$ is a subgroup of $Q_{2^{n}}$ and $S D_{2^{n}}$ and $Q_{8}$ contains the subgroup $\langle v\rangle$ of $C$. But then $x$ inverts $\langle v\rangle$ and $\langle a\rangle$ (see above) and so all eight elements in $\langle a, v\rangle x$ from $H-(Q C)$ are of order 4 , a contradiction. Indeed, we compute for any integers $i, j$ :

$$
\left(a^{i} v^{j} x\right)^{2}=a^{i} v^{j} x a^{i} v^{j} x=a^{i} v^{j} x^{2}\left(a^{i} v^{j}\right)^{x}=a^{i} v^{j} z a^{-i} v^{-j}=z .
$$

Finally, suppose that $\langle x, C\rangle \cong D_{2^{n}}, n \geq 3$, where $x$ is an involution. But then all elements in $\langle a, C\rangle x$ from $H-(Q C)$ are involutions since $x$ inverts $\langle a\rangle$ and $C$ and all other elements in $(Q C-\langle a, C\rangle) x$ from $H-(Q C)$ are elements of order 8 , a contradiction (since $H-S$ does not contain any elements of order $4)$. Indeed, we set $C=\langle c\rangle$ and we know that $b^{x}=a b$ so that for any integers $i, j$ we compute (noting that in $Q$ we have $b a^{i}=a^{i} b z^{i}$ and $b a b=a$ ):

$$
\begin{gathered}
\left(b a^{i} c^{j} x\right)^{2}=b a^{i} c^{j}\left(b a^{i} c^{j}\right)^{x}=b a^{i} c^{j} \cdot a b a^{-i} c^{-j}=a^{i} b z^{i} c^{j} a b a^{-i} c^{-j}= \\
a^{i} z^{i}(b a b) c^{j} a^{-i} c^{-j}=a^{i} z^{i} \cdot a \cdot c^{j} a^{-i} c^{-j}=z^{i} a,
\end{gathered}
$$

which is an element of order 4. Hence, all elements $b a^{i} c^{j} x$ are of order 8 , as claimed. Our lemma is proved.

In the next three lemmas we assume in addition that $Q_{8}$ is not a subgroup of $H$.

LEMMA 2.3. Assuming that $Q_{8}$ is not a subgroup of $H$, we have $\left|H: N_{H}(X)\right| \leq 2$ for each cyclic subgroup $X$ of order 4 in $H$.

Proof. Suppose that the lemma is false. Then there is a cyclic subgroup $U_{1}$ of order 4 in $H$ such that $K=N_{H}\left(U_{1}\right)$ is of index 4 in $H$. Let $M$ be a maximal subgroup of $H$ containing $K$ so that $|H: M|=|M: K|=2$ and let $m \in M-K$. Then $U_{2}=U_{1}^{m} \neq U_{1}, N_{H}\left(U_{2}\right)=K$ and so $A=U_{1} U_{2} \cong C_{4} \times C_{2}$ and $A$ is normal in $M$ (Lemma 2.1). Let $x \in H-M$ so that $A^{x} \neq A$ since $c_{2}(A)=2$ (and so $A$ cannot be normal in $H$ ) and $A^{x} \leq M$. We have $c_{2}(M) \in\{3,4,5\}$ because there must exist elements of order 4 in $H-M$. If $c_{2}(M)$ is odd, then $M$ (of order $\geq 2^{4}$ ) is of maximal class, a contradiction (since $M$ possesses an abelian subgroup of type $(4,2)$ ). Hence $c_{2}(M)=4$.

Suppose that $|M|>2^{4}$. If $\left|\Omega_{2}(M)\right|>2^{4}$, then $U_{1}$ is normal in $M$ (see [3, Introduction]), contrary to the fact that $N_{H}\left(U_{1}\right)=K<M$. Hence we must have $\left|\Omega_{2}(M)\right|=2^{4}$. In that case we may use Janko [4, Theorems 3.1, 3.3, and 3.4] since $Q_{8}$ is not a subgroup of $M$ and $c_{2}\left(\Omega_{2}(M)\right)=4$. This implies that $\Omega_{2}(M)$ is abelian of type $(4,2,2)$ and there is a cyclic subgroup of order 4 which is normal in $M$. This is a contradiction since $\Omega_{2}(M)=A A^{x}$ and so all four cyclic subgroups of order 4 in $M$ are conjugate in $H$ and so no one of them could be normal in $M$.

We have proved that $|M|=2^{4}$ so that $K=A=N_{H}\left(U_{1}\right), A A^{x}=M$ is of order $2^{4}$ and $|H|=2^{5}$. In this case $A$ and $A^{x}$ are two distinct abelian maximal subgroups of $M$ which implies $|Z(M)|=4,\left|M^{\prime}\right|=2$, the class of 
$M$ is 2 and $M$ is of exponent 4 . Suppose that $M$ is not minimal nonabelian. Then $M$ possesses a subgroup $D \cong D_{8}$ and since $M$ is not of maximal class, we have $C_{M}(D) \not \leq D$ (see [3, Proposition 1.9]). Since $c_{2}(M)=4$, we get $M=D * C$ with $C \cong C_{4}$ and $D \cap C=Z(D)$. But $D_{8} * C_{4} \cong Q_{8} * C_{4}$, contrary to our assumption. Hence $M$ is minimal nonabelian. If $M$ is metacyclic, then $M$ possesses a cyclic normal subgroup of order 4 which contradicts the fact that all four cyclic subgroups of order 4 in $M$ are conjugate in $H$. Hence $M$ is a uniquely determined nonmetacyclic minimal nonabelian group of order $2^{4}$ and exponent 4 (see [4, Proposition 1.3]).

Since $N_{H}(X)<M$ for each cyclic subgroup $X$ of order 4 in $M$, there are no elements of order 8 in $H-M$. It follows that $H-M$ consists of four elements of order 4 and 12 involutions. Set $E=\Omega_{1}(M)$ so that $E$ is elementary abelian of order $8, Z(M)=\Phi(M) \cong E_{4}$ and $Z(H) \leq Z(M)$. Let $v$ be an element of order 4 in $H-M$ so that $v^{2} \in E$ and $C_{E}(v) \cong E_{4}$ since $H-M$ contains exactly four elements of order 4 (and they are all contained in $(E\langle v\rangle)-E)$. All eight elements in $H-(M \cup E\langle v\rangle)$ are involutions and so if $u \in H-(M \cup E\langle v\rangle)$, then $u$ centralizes $E$ and $F=E \times\langle u\rangle \cong E_{16}$. In particular, $Z(M)<E<F$ and so $Z(M)=Z(H)$. Let $y \in M-E$ and we know that all cyclic subgroups of order 4 in $M$ are conjugate in $H$ to $\langle y\rangle$. But $y^{2} \in \Phi(M)=Z(H)$ and so $\mho_{1}(M)=\left\langle y^{2}\right\rangle$, contrary to $\Phi(M) \cong E_{4}$. Our lemma is proved.

Lemma 2.4. Assuming that $Q_{8}$ is not a subgroup of $H$, we have $\left|H: N_{H}(X)\right|=2$ for each cyclic subgroup $X$ of order 4 in $H$.

Proof. Suppose that the lemma is false. Then there are at least two distinct cyclic subgroups $U_{1}$ and $U_{2}$ which are normal in $H$. Let $\left\{U_{1}, U_{2}, \ldots, U_{6}\right\}$ be the set of six cyclic subgroups of $H$. Since each $U_{i}, i=1,2, \ldots, 6$, normalizes $U_{1}$ and $U_{2}$, it follows (Lemma 2.1) that $A=\left\langle U_{1}, U_{2}\right\rangle \cong C_{4} \times C_{2}$ and $A \leq Z(H)$. For each $U_{j}, j=3, \ldots, 6$, we have $\left\langle U_{1}, U_{j}\right\rangle \cong\left\langle U_{2}, U_{j}\right\rangle \cong C_{4} \times C_{2}$ and so $U_{1} \cap U_{j}=U_{2} \cap U_{j}=U_{1} \cap U_{2}=\langle z\rangle=\mho_{1}(A)$. It follows that $B=A U_{3}=\left\langle U_{1}, U_{2}, U_{3}\right\rangle$ is abelian of order $2^{4}$ and exponent 4 with $\mho_{1}(B)=\langle z\rangle$ and so $B$ is abelian of type $(4,2,2)$. Since $c_{2}(B)=4$, we nay assume that $\left\{U_{1}, U_{2}, U_{3}, U_{4}\right\}$ is the set of cyclic subgroups of order 4 in $B$. Similarly, $C=A U_{5}$ is abelian of type $(4,2,2)$ with $\mho_{1}(C)=\langle z\rangle$ so that $\left\{U_{1}, U_{2}, U_{5}, U_{6}\right\}$ is the set of cyclic subgroups of order 4 in $C$. We have $B \cap C=A$ and $H=\langle B, C\rangle$. Thus, $H / A$ is generated with two distinct involutions $(B / A)^{\sharp}$ and $(C / A)^{\sharp}$ and so $H / A \cong E_{4}$ or $H / A \cong D_{2^{n}}, n \geq 3$. In particular, $B$ and $C$ are not conjugate in $H$. Let $t$ be an involution in $H-(B \cup C)$ and let $v$ be an element of order 4 in $A \leq Z(H)$. Then $t v$ is an element of order 4 in $H-(B \cup C)$, a contradiction. Hence, all elements in $H-(B \cup C)$ are of order $\geq 8$. This implies that $B$ and $C$ are normal in $H$ and so $H=\langle B, C\rangle=B C$ is of order $2^{5}$ with two distinct abelian maximal subgroups $B$ and $C$. It follows that $\left|H^{\prime}\right| \leq 2$ and so $H$ is of class $\leq 2$. 
But $H$ is generated by its elements of order 4 and so $H$ is of exponent 4 , a contradiction.

LEMMA 2.5. Assuming that $Q_{8}$ is not a subgroup of $H$, we have $c_{2}\left(N_{H}(X)\right)=2$ for each cyclic subgroup $X$ of order 4 in $H$.

Proof. Let $U_{1}$ be a cyclic subgroup of order 4 in $H$ so that $\left|H: N_{H}\left(U_{1}\right)\right|=2$ (Lemma 2.4). Set $M=N_{H}\left(U_{1}\right)$ and taking an element $h \in H-M$ we get $U_{2}=U_{1}^{h} \neq U_{1}, N_{H}\left(U_{2}\right)=M, A=\left\langle U_{1}, U_{2}\right\rangle \cong C_{4} \times C_{2}$ (Lemma 2.1), and $A$ is normal in $H$. Assume that $M$ possesses a further cyclic subgroup $U_{3} \not \leq A$ of order 4 so that $\left\langle U_{1}, U_{3}\right\rangle \cong\left\langle U_{2}, U_{3}\right\rangle \cong C_{4} \times C_{2}$ and therefore $B=\left\langle U_{1}, U_{2}, U_{3}\right\rangle$ is abelian of type $(4,2,2)$. Since $c_{2}(B)=4$, we may assume that $\left\{U_{1}, U_{2}, U_{3}, U_{4}\right\}$ is the set of all cyclic subgroups of order 4 in $B$. There is an element $g$ of order 4 in $H-M$ and since $\left|H: N_{H}(\langle g\rangle)\right|=2$, $U_{5}=\langle g\rangle$ and $U_{6}=\left\langle g^{x}\right\rangle$ (with an $x \in H-N_{H}(\langle g\rangle)$ ) give two last cyclic subgroups of order 4 in $H$ which give exactly four elements of order 4 in $H-M$. This implies that $B=\Omega_{2}^{*}(M)$ is normal in $H$ and $U_{3}$ and $U_{4}$ are conjugate in $H$.

Set $H_{0}=B U_{5}$. If $c_{2}\left(H_{0}\right)=5$, then $H_{0}$ is of maximal class, a contradiction. Hence $c_{2}\left(H_{0}\right)=6$ and so $H_{0}=B U_{5}=H$. Set $B_{0}=\Omega_{1}(B) \cong E_{8}$. Suppose $B \cap U_{5}=\{1\}$ so that $|H: B|=4$. Since $\left|H: N_{H}\left(U_{5}\right)\right|=2, U_{5}$ centralizes a four-subgroup $S$ in $B_{0}$. But then all eight elements of order 4 in $S \times U_{5}$ lie in $H-B$, a contradiction. Hence $B \cap U_{5} \cong C_{2}$ and so $|H: B|=2$, $|H|=2^{5}, B=M$, and $N_{H}\left(U_{3}\right)=N_{H}\left(U_{4}\right)=B$. This implies that there are no elements of order 8 in $H-B$ and so $H-B$ consists of four elements of order 4 and twelve involutions.

We have $\left|B: N_{B}(\langle g\rangle)\right|=2$, where $\langle g\rangle=U_{5}$ and $N_{B}(\langle g\rangle)$ cannot contain an element $x$ of order 4 (otherwise, that element $x$ would centralize $U_{5}$, contrary to the fact that $\left.C_{H}(x)=B\right)$. Hence $N_{B}(\langle g\rangle)=B_{0}$. If $g$ centralizes $B_{0}$, then there are eight elements of order 4 in $H-B$, a contradiction. Hence $C_{B}(g)=C_{B_{0}}(g)=Z \cong E_{4}$ and so $Z(H)=Z$. The set $B_{0} g$ consists of four elements of order 4 and four involutions. Hence all eight elements in $H-\left(B \cup B_{0}\langle g\rangle\right)$ are involutions and if $t$ is one of them, then $H-B=B_{0} g \cup B_{0} t$ and $B_{0} g \cap B_{0} t=\emptyset$ so that $t$ must centralize $B_{0}$ and therefore $B_{0} \leq Z(H)$, contrary to the fact that $Z(H)=Z \cong E_{4}$. Our lemma is proved.

THEOREM 2.6. Let $G$ be a 2-group with exactly six cyclic subgroups of order 4 and let $H=\Omega_{2}^{*}(G)=\langle x \in G \mid o(x)=4\rangle$ be of order $>2^{4}$. Then $H$ is of order $2^{5}$ and we have the following three possibilities:

(a) $H \cong Q_{8} * Q_{8}$ is extraspecial (of type "+");

(b) $H \cong Q_{16} * C_{4}$ with $Q_{16} \cap C_{4}=Z\left(Q_{16}\right)$;

(c) $H$ is a special group possessing a unique elementary abelian subgroup $E$ of order $2^{4}$ and there is an involution $t \in H-E$ such that $H=\langle E, t\rangle$ and $C_{E}(t)=Z(H) \cong E_{4}$. 
Proof. In view of Lemma 2.2, we may assume that $Q_{8}$ is not a subgroup of $H$ and so we may use Lemmas 2.1,2.4, and 2.5. Let $U_{1}$ be a cyclic subgroup of order 4 in $H$. Set $K=N_{H}\left(U_{1}\right)$ so that $|H: K|=2$ and if $h \in H-K$, then $U_{2}=U_{1}^{h} \neq U_{1}, A=\left\langle U_{1}, U_{2}\right\rangle=\Omega_{2}^{*}(K) \cong C_{4} \times C_{2}$ is normal in $H$, $N_{H}\left(U_{2}\right)=K$ and so no one of $U_{1}, U_{2}$ is characteristic in $K$. Note that $|H|>2^{4}$ and so $|K|>2^{3}$.

We are in a position to use Proposition 1.1 which gives that $K$ is a uniquely determined group of order $2^{5}$ or $2^{4}$. We may assume that we have the following conjugacy classes of our six cyclic subgroups of order 4 in $H: U_{1} \sim U_{2}$, $U_{3} \sim U_{4}$, and $U_{5} \sim U_{6}$. Assume that $|K|=2^{5}$ in which case $|H|=2^{6}$. It follows that $\Phi\left(N_{H}\left(U_{1}\right)\right)=\left\langle U_{1}, U_{2}\right\rangle$ and similarly (since $\left|H: N_{H}\left(U_{3}\right)\right|=$ $\left.\left|H: N_{H}\left(U_{5}\right)\right|=2\right), N_{H}\left(U_{3}\right) \cong N_{H}\left(U_{5}\right) \cong K$. But then $\Phi\left(N_{H}\left(U_{3}\right)\right)=$ $\left\langle U_{3}, U_{4}\right\rangle, \Phi\left(N_{H}\left(U_{5}\right)\right)=\left\langle U_{5}, U_{6}\right\rangle$ and therefore $\Phi(H) \geq\left\langle U_{1}, U_{2}, U_{3}, U_{4}\right\rangle=H$, a contradiction.

We have proved that $K=N_{H}\left(U_{1}\right)=N_{H}\left(U_{2}\right)$ is of order $2^{4}$ and then $K \cong D_{8} \times C_{2}$ (Proposition 1.1(i)) and $|H|=2^{5}$. The subgroup $K$ has exactly three abelian maximal subgroups: $F_{1} \cong E_{8}, F_{2} \cong E_{8}$, and $A=\left\langle U_{1}, U_{2}\right\rangle \cong$ $C_{4} \times C_{2}$, where $F_{1} \cap F_{2}=F_{0}=Z(K) \cong E_{4}$. There are no elements of order 8 in $H-K$ since $N_{H}\left(U_{1}\right)=N_{H}\left(U_{2}\right)=K$ and so $H-K$ consists of eight elements of order 4 and eight involutions. Let $r$ be an involution in $H-K$. Then $r k(k \in K)$ is an involution if and only if $k^{r}=k^{-1}$. But $U_{1}^{r}=U_{2}$ and so the fact that $H-K$ contains exactly eight involutions gives at once that $C_{K}(r)$ is elementary abelian of order 8 and therefore we may assume that $C_{K}(r)=F_{1}$. Indeed, the fact that $U_{1}^{r}=U_{2}$ implies that $r$ neither centralizes nor inverts any of the four elements of order 4 in $K-\left(F_{1} \cup F_{2}\right)$. Since $H-K$ contains exactly eight involutions, it follows that $r$ inverts (and therefore centralizes) the element 1 and seven further involutions in $K$ and so in $F_{1} \cup F_{2}$. Note that no involution in $F_{1}-F_{0}$ commutes with any involution in $F_{2}-F_{0}$ (otherwise, $K$ would be abelian!). If all these seven involutions are not contained in $F_{1}$ or in $F_{2}$, then $r$ centralizes an involution $t_{1} \in F_{1}-F_{0}$ and an involution $t_{2} \in F_{2}-F_{0}$ and so $r$ centralizes $t_{1} t_{2}$. But $t_{1}$ and $t_{2}$ do not commute and so $r$ centralizes the element $t_{1} t_{2}$ of order 4 , a contradiction. The subgroup $E=F_{1} \times\langle r\rangle$ is a unique elementary abelian subgroup of order 16 in $H$ and for each involution $x \in E-F_{1}, C_{K}(x)=F_{1}$ and so if $t$ is an involution in $F_{2}-F_{0}$, then $C_{E}(t)=C_{F_{1}}(t)=F_{0}=Z(K)=Z(H) \cong E_{4}$. We have obtained the group (c) stated in our theorem.

THEOREM 2.7. Let $G$ be a 2-group with exactly six cyclic subgroups of order 4 and let $H=\Omega_{2}^{*}(G)$ be of order $>2^{4}$. Then $H$ is of order $2^{5}$ and we have three possibilities for the structure of $H$ (Theorem 2.6). However, if $G>H$, then $H \cong Q_{16} * C_{4},|G: H|=2,|G|=2^{6}$, and we have the following two possibilities: 
(i) $G$ has a dihedral subgroup $D=\left\langle f, \xi \mid f^{16}=\xi^{2}=1, f^{\xi}=f^{-1}\right\rangle \cong D_{16}$ of index 2 and an involution $u \in G-D$ so that $[u, \xi]=1$ and $f^{u}=f z$, $z=f^{8}$.

(ii)

$$
\begin{gathered}
G=\langle a, t| a^{16}=t^{2}=1, a^{8}=z, a^{4}=v, a^{t}=a^{-1} v u, u^{2}=1, \\
\left.[u, a]=1, u^{t}=u z\right\rangle,
\end{gathered}
$$

where $G$ is a $U_{2}$-group with respect to $U=\langle u, z\rangle \cong E_{4}, G / U \cong S D_{16}$ and $Z(G)=\langle u v\rangle \cong C_{4}$.

Proof. For the structure of $H=\Omega_{2}^{*}(G)$ we use Theorem 2.6. We assume in addition that $G>H$. If $H \cong Q_{8} * Q_{8}$ (Theorem 2.6(a)), then we have a contradiction by [5, Theorem 2].

Suppose that $H$ is a special group given in Theorem 2.6(c). Let $L$ be a subgroup of $G$ containing $H$ so that $|L: H|=2$. Let $E$ be a unique elementary abelian subgroup of order 16 in $H$ so that $H$ is normal in $L$. Let $j$ be an involution in $H-E$ so that $C_{E}(j)=E_{0}=Z(H) \cong E_{4}$ is normal in $L$ and $F=E_{0} \times\langle j\rangle=C_{H}(j) \cong E_{8}$ is normal in $L$ since 12 elements in $H-(E \cup F)$ are of order 4 . Four involutions in $F-E_{0}$ form a single conjugate class in $H$ and so $I=C_{L}(j)$ covers $L / H$ and $I \cap H=F$. Since there are no elements of order 4 in $L-H$, all elements in $I-F$ must be involutions and therefore $I \cong E_{16}$ and $E_{0} \leq Z(L)$. Let $i$ be an involution in $I-F$ and consider the subgroup $J=E\langle i\rangle$ of order $2^{5}$, where $J \cap H=E$. Again, all elements in $J-E$ must be involutions and so $J \cong E_{32}$. We get $C_{H}(i) \geq\langle E, F\rangle=H$. If $v$ is an element of order 4 in $H$, then $v i$ is of order 4 and $v i \in L-H$, a contradiction.

We have proved that $H \cong Q_{16} * C_{4}$ must be a group given in Theorem 2.6(b). We may set $H=Q * C$, where

$$
Q=\left\langle b, t \mid b^{8}=1, t^{2}=b^{4}=z, b^{t}=b^{-1}\right\rangle \cong Q_{16}, \quad C=\langle v\rangle \cong C_{4}, \quad v^{2}=z,
$$

and $Q \cap C=\langle z\rangle$. The subgroup $Q$ is generated by all (five) noncentral cyclic subgroups of order 4 in $H$ and so $Q$ is normal in $G$. Set $D=C_{G}(Q)$ so that $D \geq C$ and $D \cap H=C$. If there is an involution $i \in D-C$, then $o\left(b^{2} i\right)=4$ and $b^{2} i \notin H$, a contradiction. Hence $z$ is a unique involution in $D$. Since $c_{2}(D)=1, D$ cannot be generalized quaternion and so $D$ is cyclic. Let $d \in D-C$ be an element of order 8 . Then $b^{4}=d^{4}=z$ and so $o(b d)=4$ with $b d \notin H$, a contradiction. We have proved that $D=C=C_{G}(Q)$.

The automorphism group $\operatorname{Aut}(Q)$ is generated by $\operatorname{Inn}(Q) \cong D_{8}$ and two involutory outer automorphisms $\alpha$ and $\beta$ induced by $t^{\alpha}=t b, b^{\alpha}=b^{-1}$, $t^{\beta}=t, b^{\beta}=b z$, where $[\alpha, \beta]=i_{b^{2}}$ (the inner automorphism of $Q$ induced by conjugation with the element $b^{2}$ ) and so $\operatorname{Aut}(Q) / \operatorname{Inn}(Q) \cong E_{4}$ (and in fact $\left.\langle\alpha, \beta\rangle \cong D_{8}\right)$. The subgroup $Q$ contains exactly two quaternion subgroups $Q_{1}$ and $Q_{2}$ and we have $Q_{1}^{\beta}=Q_{1}, Q_{2}^{\beta}=Q_{2}$, and $Q_{1}^{\alpha}=Q_{2}$. It follows that $G / H \neq\{1\}$ is elementary abelian of order $\leq 4$. 
Assume that $L=N_{G}\left(Q_{1}\right)>H$ so that $|L: H|=2$. Since $Q /\langle z\rangle \cong D_{8}$ is isomorphic to an $S_{2}$-subgroup of $\operatorname{Aut}\left(Q_{1}\right) \cong S_{4}$, it follows that $C_{0}=$ $C_{L}\left(Q_{1}\right)>C$ and $\left|C_{0}: C\right|=2$. If $y \in C_{0}-C$ is an involution, then $o\left(b^{2} y\right)=4$ (because $\left.b^{2} \in Q_{1}\right)$ and $b^{2} y \notin H$, a contradiction. Since $c_{2}\left(C_{0}\right)=1$, we get that $C_{0}=\langle c\rangle \cong C_{8}$ is cyclic with $c^{4}=z$. Now, $c$ normalizes $\langle b\rangle$ (since $Q$ is normal in $G$ and $\langle b\rangle$ is a unique cyclic subgroup of index 2 in $Q$ ) and centralizes $\left\langle b^{2}\right\rangle=\langle b\rangle \cap Q_{1}$, but $c$ does not centralize $\langle b\rangle$ (otherwise, $c$ would centralize $\left\langle b, Q_{1}\right\rangle=Q$, a contradiction) and so we get $b^{c}=b z,\langle b, c\rangle^{\prime}=\langle z\rangle$, class of $\langle b, c\rangle$ is $2,(b c)^{4}=b^{4} c^{4}[c, b]^{6}=z z z^{6}=1, o(b c)=4$, and $b c \notin H$, a contradiction.

We have proved that $|G / H|=2,|G|=2^{6}$, and if $g \in G-H$, then $Q_{1}^{g}=Q_{2}$. In particular, $C_{G}(t)=\langle t, v\rangle \cong C_{4} \times C_{2}$ and so eight elements of order 4 in $Q-\langle b\rangle$ form a single conjugate class in $G$. Set $T=\langle b\rangle\langle v\rangle \cong C_{8} \times C_{2}$ which is normal in $G$ and eight elements in $H-(Q \cup T)$ are involutions which form a single conjugate class in $G$ and so if $t v$ is one of them, then $C_{G}(t v)=\langle t, v\rangle$. In particular, if $x \in G-H$, then $x^{2} \in T$. We have $U=$ $\Omega_{1}(T)=\left\langle z, b^{2} v\right\rangle \cong E_{4}$ is normal in $G, \Omega_{2}(T)=\left\langle b^{2}, v\right\rangle \cong C_{4} \times C_{2}$, and $\left\langle b^{2}\right\rangle$ and $\langle v\rangle$ are normal in $G$.

Suppose that there is an involution $\xi \in G-H$. Then $\xi$ inverts $\langle v\rangle$ and $\left\langle b^{2}\right\rangle$ (otherwise, $\xi$ centralizes $v$ or $b^{2}$ and then $\xi v$ or $\xi b^{2}$ would be an element of order 4 in $G-H$, a contradiction). If $b^{\xi}=b^{-1} z$, then $(\xi b)^{2}=b^{\xi} b=z$ and $o(\xi b)=4$ with $\xi b \notin H$, a contradiction. Hence $\xi$ inverts each element in $T$ and so, in particular, $\xi$ centralizes $U$. Since $Q_{1}^{\xi}=Q_{2}$, we have $t^{\xi}=t b^{i}$, where $i$ is odd. Set $b^{2} v=u$ and $\xi t=f$ so that $\xi$ centralizes the involution $u$,

$$
\begin{gathered}
f^{2}=\xi t \xi t=t^{\xi} t=\left(b^{i}\right)^{t}=b^{-i}, o(f)=16, f^{8}=\left(b^{-i}\right)^{4}=z, \\
f^{\xi}=(\xi t)^{\xi}=\xi t^{\xi}=\xi t b^{i}=f b^{i}=f^{-1}\left(f^{2} b^{i}\right)=f^{-1} b^{-i} b^{i}=f^{-1},\langle f, \xi\rangle \cong D_{16}
\end{gathered}
$$

and

$$
\begin{gathered}
f^{u}=(\xi t)^{b^{2} v}=v^{-1} b^{-2} \xi t b^{2} v=\xi\left(v^{-1} b^{-2}\right)^{\xi} t b^{2} v=\xi v b^{2} t b^{2} v= \\
(\xi t)\left(v b^{2}\right)^{t} b^{2} v=(\xi t) v b^{-2} b^{2} v=\xi t v^{2}=f z .
\end{gathered}
$$

We have obtained the group given in part (i) of our theorem.

It remains to investigate the case, where there are no involutions in $G-H$. Then 32 elements in $G-H$ are of order 8 or 16. If all 32 elements in $G-H$ are of order 8 , then $c_{3}(G)=10$ and therefore $G$ is a $U_{2}$-group (see section $1)$. But then $G$ must also have elements of order 16 which is not the case. If all 32 elements in $G-H$ are of order 16, then $c_{4}(G)=4$ and $c_{3}(G)=2$. Again, $G$ is a $U_{2}$-group. But a $U_{2}$-group of order $2^{6}$ has exactly two cyclic subgroups of order 16 , a contradiction. Hence $G-H$ contains elements of order 8 and 16. Since the number of cyclic subgroups of order 16 must be even (otherwise, $G$ would be of maximal class), it follows that $G-H$ has exactly 16 elements of order 16 (and so $c_{4}(G)=2$ ) and exactly 16 elements of order 8 . Hence $c_{3}(G)=6$ and so $G$ is a $U_{2}$-group with respect to $U$ since in a $U_{2}$-group a normal four-subgroup is unique. If $R / U$ is a cyclic subgroup of 
index 2 in $G / U$, then $G-R$ contains exactly eight involutions, eight elements of order 4 , and 16 elements of order 8 . Hence $G / U \cong S D_{16}$ and $\Phi(R) \cong C_{8}$. Since $H$ is nonmetacyclic, $G$ is also nonmetacyclic. We have $\Phi(G) \leq T$ and so there are exactly three maximal subgroups of $G$ containing $T$. They are $H, R$ and a certain subgroup $V$ with the property that all 16 elements in $V-T$ are of order 8. Since $\Omega_{2}(V)=\Omega_{2}(T)=\left\langle b^{2}, v\right\rangle \cong C_{4} \times C_{2},|V|=2^{5}$, and $V$ has no elements of order 16, $V$ must be isomorphic to a group (d) given in [4, Proposition 1.4] and so $\Phi(V)=\Omega_{2}(V)$ and $Z(V) \cong C_{4}$. We get $\Phi(G) \geq\langle\Phi(R), \Phi(V)\rangle=T$ and so $G$ is 2-generated, i.e., $d(G)=2$. Also, $Z(V) \cong C_{4}$ implies that $U \not \leq Z(V)$ and so $C_{G}(U)=R$ (because $C_{G}(U)$ must be a maximal subgroup of $G$ containing $T$ and also $U \not Z Z(H)$ ). Since $\Phi(T)=\left\langle b^{2}\right\rangle$ and $\Phi(V)=\left\langle b^{2}, v\right\rangle$ (and no involution in $\Phi(V)-\langle z\rangle$ could be a square of an element in $V-T$ because $U \notin Z(V))$, there is an element $s \in V-T$ such that $s^{2}=v$. Hence, $C_{G}(v) \geq\langle H, s\rangle=G$ and so $Z(G) \cong C_{4}$. We have obtained a nonmetacyclic $U_{2}$-group $G$ of order $2^{6}$ with respect to $U \cong E_{4}$ such that $G / U \cong S D_{16}, d(G)=2$, and $Z(G) \cong C_{4}$. It follows that $G$ must be isomorphic to a $U_{2}$-group given in [3, Theorem $\left.6.3(\mathrm{c})\right]$. We have obtained the group given in part (ii) of our theorem.

\section{2-GROUPS WITH ONE CONJUGACY CLASS OF CYCLIC SUBGROUPS OF ORDER 4}

THEOREM 3.1. Let $G$ be a 2-group of exponent $>2$ all of whose cyclic subgroups of order 4 are conjugate. Then $G$ has exactly one cyclic subgroup of order 4 and $G$ is either cyclic or dihedral.

Proof. First suppose that $G$ has more than one cyclic subgroup of order 4. Let $U$ be one of them and set $K=N_{G}(U)$ so that $|G: K| \geq 2$ and let $M$ be a maximal subgroup of $G$ containing $K$. Then each cyclic subgroup of order 4 is contained in $M$ and if $X$ is one of them, then $N_{G}(X) \leq M$ (since $X$ is conjugate in $G$ to $U$ ). Let $x$ be any element in $G-M$. We know that $x$ is not of order 4 and suppose that $o(x) \geq 8$. But then $x^{2} \in M$ and $o\left(x^{2}\right) \geq 4$ and so $x$ centralizes a cyclic subgroup of order 4 in $M$, a contradiction. Hence each element $x$ in $G-M$ is an involution and so $M$ must be abelian and $x$ acts invertingly on $M$. But then $U$ is normal in $G$, a contradiction.

We have proved that $G$ has a unique cyclic subgroup $V=\langle v\rangle$ of order 4 so that $V$ is normal in $G$. Then our result follows by [2, Theorem 1.17]. Here we give also a direct proof. Set $C=C_{G}(V)$ and we have $|G: C| \leq 2$. If $C$ possesses an involution $t \neq v^{2}$, then $\langle t v\rangle$ is a cyclic subgroup of order 4 distinct from $V$, a contradiction. It follows that $C$ has the unique involution $v^{2}$ and so $C$ is cyclic. If $|G: C|=2$, then $G$ is dihedral and we are done. 


\section{REFERENCES}

[1] Y. Berkovich, On the number of elements of given order in a finite p-group, Israel J. Math. 73 (1991), 107-112.

[2] Y. Berkovich, Groups of prime power order, Parts I, II, and III (with Z. Janko), in preparation.

[3] Z. Janko, Finite 2-groups with exactly four cyclic subgroups of order $2^{n}$, J.reine angew. Math. 566 (2004), 135-181.

[4] Z. Janko, Finite 2-groups $G$ with $\left|\Omega_{2}(G)\right|=16$, Glas. Mat. Ser. III 40 (2005), 71-86.

[5] Z. Janko, Elements of order at most 4 in finite 2-groups, 2, J. Group Theory 8 (2005), 683-686.

[6] Z. Janko, Finite 2-groups with exactly one nonmetacyclic maximal subgroup, submitted.

Z. Janko

Mathematical Institute

University of Heidelberg

69120 Heidelberg

Germany

E-mail: janko@mathi.uni-heidelberg.de

Received: 27.9.2006. 